\title{
Survey on Smart Reader for Blind and Visually Impaired (BVI)
}

\author{
Geetha M.N.*, Sheethal H.V., Sindhu S., Ayesha Siddiqa J. and Chandan H.C. \\ Department of Electronics and Communication Engineering, Vidyavardhaka College of Engineering, Mysuru \\ 570002, India; geetha.mn@vvce.ac.in, hvsheethal1997@gamil.com, ssindhu807@gmail.com, \\ siddiqaaa38@gmail.com, chandanshetty1996@gmail.com
}

\begin{abstract}
Objectives: To make a survey on Smart readers for Blind and visually impaired people and propose a suitable approach. Methods analysis: Visually impaired people need special systems like Braille for reading and writing for the blind. They also find it difficult to use the latest gadgets like smartphones or computers unless some specific modification is done to these devices which will assist them. The development of smart devices will significantly enhance their livelihood. Various smart devices have been developed where Optical Character Recognition and Text to Speech is used to capture the written or printed text and convert it into audio signals. There are many drawbacks to all these devices. Here we propose a new Smart Reader which will overcome some of these drawbacks. Findings/application: This system aims to develop costeffective assistive technologies for blind people and provide a greater degree of independence in their daily activities. The main advantage of this research is to detect the text and give the voice output for visually impaired. It will help the blind to read and acquire knowledge from all the printed material without converting the same into Braille format.
\end{abstract}

Keywords: Blind and Visually Impaired (BVI), Optical Character Recognition, Text to Speech, Raspberry Pi

\section{Introduction}

According to the World Health Organisation (WHO), 250 million people are estimated to be blind or partially blind. $90 \%$ of visually impaired people live in developing countries. Books and all the printed material are the main sources of knowledge. The blind or visually impaired people cannot access this knowledge. Braille is a writing and reading system used by the blind. However, converting all the available knowledge into Braille will be a tedious task. 1 New smart devices for the reading of the printed text need to be developed for the blind which is readily available and affordable to the blind community. Here we do a survey of the existing research work on the various smart devices developed for the blind for reading. We also propose a new idea for a smart reader that can easily read the printed text or document. The scope of this research is to provide a solution and to help the blind or visually impaired people access the document easily and they can make use of this device to enhance their knowledge.

\section{Literature Survey}

Many new devices have been developed to assist the blind to overcome their disability. $\underline{2}$ Some of the recent developments in assistive technology for the blind is described here. A Screen Reader uses a text to speech engine to convert the text displayed on the screen to audible signals. The text to speech software is embedded with the hardware which converts the displayed text into speech in the corresponding language.,, 4 Another device called the Finger Reader also helps the blind in the reading of texts from the printed material. This is a device worn on the finger. The visually impaired person wearing this device scans the text line by line with their finger and the text is converted to audio signals by a text to a speech synthesizer. This device also has a sensor to alert the

${ }^{*}$ Author for correspondence 
reader if the finger moves from one line to another so it helps the reader to scan in a straight line. $\frac{5}{\mathrm{E}}$-book Readers are another device that is commonly available where the e-book is read aloud using the text to speech engine. ${ }^{6} \mathrm{Few}$ studies where the researchers have developed devices which captures the images of the text written in a printed book using an Optical Character Recognition (OCR) engine and converts this into a speech using a text to speech conversion unit on a Raspberry Pi board. $\underline{-}^{-12}$

The existing technologies for visually impaired people have many drawbacks for accessing printed text with problems such as alignment, focus, accuracy, mobility and efficiency. This is a device that assists blind people to read printed text. A real-time image of the text is captured using the camera which is given to the main module. A Raspberry Pi board which is like a mini-computer is used as the main module. This module contains the image processing code loaded to process this image. The optical character recognition technique is the image processing code used. The image is processed internally in the Raspberry Pi hardware to separate the text from the captured image. The captured image is then separated by using the OPEN CV (Open Source Computer Vision) library. Tesseract OCR is used to identify the desired letters in the text. Optical Character Recognition (OCR) is the process of electronically converting the handwritten or printed text into machine-encoded text. A camera detects the image of the paper and the image is processed by using the open CV library. The text from the image is recognized and is converted to machine-encoded text. For text to speech application Text to Speech (TST) engine is used which converts the digital text into a high-quality voice signal. Now the converted voice is output through an earphone connected to the audio jack port. This device supports free movement, it requires minimal setup and can be used in real-time. The text which is captured from the camera is converted into a speech signal using local sequential text scanning with the help of OCR the text speech algorithm is implemented. Raspberry PI model provides page and audio control using python software. This program gives an audio signal as output. This enables visually impaired people to read this text easily.

An Electro-Mechanical System to turn the page of the book when the user requests. All are actuated by Servo motor and controlled by Raspberry Pi. Wheel with a rolling surface made of rubber. A gripper helps for friction and lift with tangential force. Rolling friction between the page and the wheel creates a gap between the pages. $\underline{12}-\underline{15}$ Here we present an idea for a Smart Book reader with OCR based image to text conversion, text to speech, dictionary query and page-turning mechanism.

\section{Block Diagram}

Figure 1 shows the block diagram of the proposed Smart Book Reader. It consists of three main parts Input, Processing unit and the Output. The input for the smart reader is via the camera, microphone and the number keypad. The image of the printed text is captured using the camera. The microphone is used for dictionary queries. The processing unit consists of the Raspberry $\mathrm{Pi}$ module. The output consists of the speaker/headphone and the page turning implement. The text is captured by a camera and the image is detected using the processor and then extracted using OCR. The extracted image is then converted into a speech signal using the Text To Speech Conversion (TTSC). The converted audio signal is read out on the speaker.

\section{Hardware Components}

1) USB Camera - as an input device for capturing the image of the book's page.

2) Raspberry Pi 3 Model B - as a processing unit.

3) Speaker/Headphone - as an output device for listening to the speech output.

4) Servo Motors - as actuators for the roller wheel, lifter and turner arms.

5) Microphone - as an input device for the dictionary query feature.

6) Push Buttons - as an interrupt button for BVI user interaction.

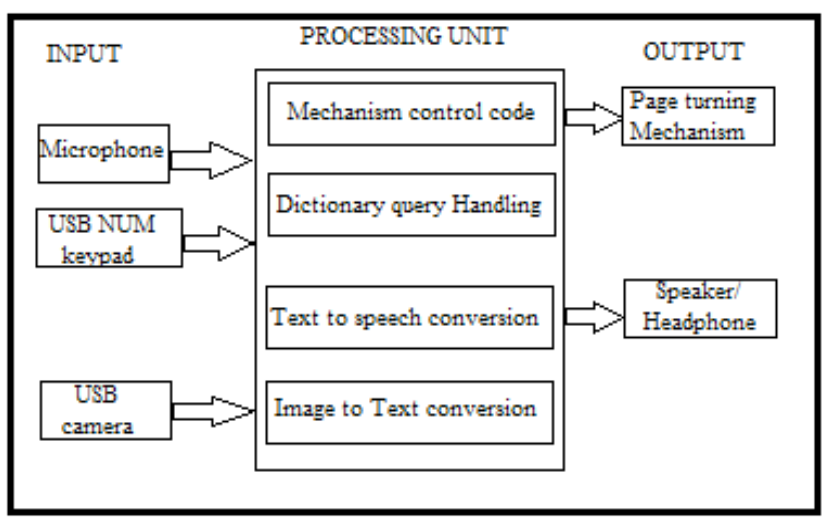

Figure 1. Block diagram of smart book reader. 
7) Bench support -rectangular plywood for hosting the mechanism, Camera, and RPI system all together tightly.

8) Book - the main source of knowledge, for capturing the image and turning mechanism.

9) Monitor - as a display device for Verification and Debugging.

\section{Raspberry Pi 3 Model B}

The heart and brain of the project design are best suited with Raspberry Pi3. It acts as a Microprocessor and Controller. Supports OS, programmable GPIO pinout

- Processor - An SoC of Broadcom BCM2387 chipset with 1.2GHz clock speed, Quad-Core ARM CortexA53, 32kB Level 1 and 512kB Level 2 cache memory, $802.11 \mathrm{~b} / \mathrm{g} / \mathrm{n}$ Wireless LAN and Bluetooth 4.1.

- GPU - Dual Core Video Core IV $^{\circledR}$ Multimedia Co-Processor.

- Memory - 1GB LPDDR2 DRAM

- Operating System - The pi boots from Micro SD card, running a Debian based OS like Raspbian,

- Power - The Pi can be easily powered up using micro USB socket $5 \mathrm{~V}, 2.5 \mathrm{~A}$

- GPIO - The Pi comes with 40-pin expansion headers arranged as 2x20 strip, providing 27 GPIO pins as well as $+3.3 \mathrm{~V},+5 \mathrm{~V}$ and GND supply lines.

\section{Programming Modules}

The raspberry pi is instructed through the Python 2.7 programming platform. The various modules installed on python are:

1) Open CV library-for image capture and preprocessing

2) Google Cloud Vision API or Python- Tesseract Image to Text conversion engine.

3) GTTS - google text to speech conversion engine.

4) Python-VLC - sound player.

5) Py Dictionary - for dictionary query feature.

6) Speech Recognition - for speech to text conversion

\section{Image to Text Conversion}

The image is first converted into a text document and this text is later converted into speech. The image is first captured using a USB camera. This image is stored on the Raspberry Pi. This image then undergoes pre-processing.
After pre-processing, the text from the image is extracted and pasted in a text document.

\section{Text to Speech Conversion}

This is done by an API called Google text to speech. This API takes in the input as a text and gives the output in the form of an audio file. This audio file is later saved as .mp3 or .m4a or any other format. This .mp3 audio file is later played with the help of an audio player, such as VLC player.

\section{Dictionary Query}

This feature helps in providing the meaning of a given word to the user. The user interrupts the system by pressing a button and speaking a word into the microphone. The system then takes in the word and converts this into text and send this text over to Web Note to fetch the meaning. This is handled by Py- Dictionary Module. This meaning is placed in a text document. This text is again converted into speech and read aloud. After this, the system resumes the previous task.

\section{Page Turning Mechanism}

The Page Turning mechanism. An Electro-Mechanical System to turn the page of the book when the user requests.

It involves three main components.

- Roller Wheel

- Lifter Arm

- Turner Arm

All are actuated by Servo motor and controlled by Raspberry Pi. Wheel with Rolling Surface made of rubber. A gripper helps for friction and lift with tangential force. Rolling friction between the page and the wheel creates a gap between the pages. Driven by a servo motor to provide a suitable angle and torque.

A Lifting Arm which lifts the Roller wheel with the appropriate angle. Gives variable normal force on the page. The angle of rotation should be increased periodically as pages get reduced. Made up of a thick stack of foam sheets attached to a servo. Rotating in a horizontal plane from 0 to 180 degrees for turning the lifted page. Positioning plays an important role, the lifting arm should be placed properly so that the error of lifting more than one page is reduced. 


\section{Applications}

- Web applications

- Email readings

- Mobile applications for intelligent speaking system

- Querying features

This system finds its interesting applications in libraries, auditorium, offices where instructions and notices are to be read.

\section{Conclusion}

A survey of existing smart reading assistance for blind and visually impaired is carried out. The existing systems have many drawbacks. Here we propose a new idea where the system provides an autonomous page turning mechanism and interactive dictionary querying feature, ultimately giving a feeling of comfort for BVI. Once developed it can act as a perfect personal device for the user. The system even finds small applications in Schools, Libraries etc.

\section{Acknowledgement}

The authors express gratitude to Accendere Knowledge Management Services Pvt Ltd for the assistance provided in preparing the manuscript.

\section{References}

1. Sojib N, Zafar Iqbal M. Single cell Bangla braille book reader for visually impaired people. In: International conference on Bangla speech and language processing; 2018. P. 1-5.

2. Singh A, Devnani S, Kushwaha V, Mishra S, Gupta A, Soundrya Pandian KK. An efficient auxiliary reading device for visually impaired. In: International conference on smart city and emerging technology; 2018.

3. Asakawa C, Takagi H, Ino S, Ifukube T. Auditory and tactile interfaces for representing the visual effects on the web. In:
Proceedings of the fifth international ACM conference on assistive technologies - assets; 2002.

4. Sandhya S, Sumithra Devi KA. Accessibility evaluation of websites using screen reader. In: 7th international conference on next generation web services practices; 2011.

5. Shilkrot R, Huber J, Mengee W, Maes P, Nanayakkara SC. Finger reader. In: Proceedings of the 33rd annual ACM conference on human factors in computing systems - CHI '15; 2015.

6. Jung J, Chan-Olmsted S, Park B, Kim Y. Factors affecting e-book reader awareness, interest, and intention to use. New Media Soc. 2012;14(2):204-24.

7. Sharma S, Bhat D, Hegde NR, Yajnesh AP, Mythri D. Drusti - a smart reader for visually impaired people. Int J Eng Res Technol. 2018;6(15):1-4.

8. Musale S, Ghiye V. Smart reader for visually impaired. In: Proceedings of the second international conference on inventive system and control; 2018.

9. Muthusenthil B, Kishore S, Joshuva J, Narendiran K. Smart assistance for blind people using raspberry pi. Int J Adv Res Ideas Innov Technol. 2018;4(2):1-8.

10. James SA, Sanjana S, Monisha M. OCR based automatic book reader for the visually impaired using raspberry pi. Int J Innov Res Comput Commun Eng. 2016;4(7):1-8.

11. Trivedi A, Pant N, Shah P, Sonik S, Agrawal S. Speech to text and text to speech recognition systems-a review. IOSR J Comput Eng. 2018;20(2):36-43.

12. Hagargund AG, Thota SV, Bera M, Shaik EF. Image to speech conversion for visually impaired. Int J Latest Res Eng Technol. 2017;3(6):1-7.

13. Deshmukh PB. Book flipping and scanning machine. Int J Innov Res Sci Eng Technol. 2016;5(10):1-4.

14. Lee J, Jeon W, Cha Y, Yang H. Automatic page-turning mechanism with near-field electroadhesive force for linearly correctable imaging. In: IEEE/RSJ international conference on intelligent robots and systems; 2017. P. 1.

15. Rajendra A.B., Rajkumar N., Bhat S.N., Suhas T.R., Joshi S.P.N. E-Learning Web Accessibility Framework for Deaf/ Blind Kannada-Speaking Disabled People. In: Singh P., Kar A., Singh Y., Kolekar M., Tanwar S. (eds) Proceedings of ICRIC 2019. Lecture Notes in Electrical Engineering, 2020; vol 597. Springer, Cham. 